\title{
Role of Nitric Oxide in Isoproterenol-Induced Myocardial Infarction
}

\author{
Victoria Chagoya de Sánchez et al.* \\ Departamento de Biología Celular y Desarrollo, \\ Instituto de Fisiología Celular, \\ Universidad Nacional Autónoma de México, \\ Mexico
}

\section{Introduction}

Myocardial infarction (MI) is an important cause of mortality around the world, resulting from an ischemic necrosis induced by a vascular occlusion. In general, MI occurs unexpectedly and the clinical syndrome previous to MI is difficult to detect. Animal models of MI are very useful in the study of prevention, diagnosis and therapy design for human MI [Smith \& Nuttall., 1985]. MI induced by ligation of the left anterior descending coronary artery is the animal model most frequently used; however, the anesthesia and surgical procedures affect the conditions in which the infarction occurred [Wang et al., 2006]. Therefore, MI in animal models studies should be induced in conscious animals with intact reflexes for greater clinical relevance. Myocardial infarction induced by isoproterenol (ISO) using toxic concentration of this $\beta$-adrenergic agonist drug, originally described by Rona [Rona et al., 1959] has been used by several groups to study the cardiotoxic effects of this molecule [Stanton et al,1969]. This model becomes relevant if we consider that increased adrenergic activation plays a role as a trigger of acute myocardial infarction. In addition, the stress associated to MI in patients, increases catecholamine blood levels, which in turn, augment contraction force of the heart and ATP utilization favoring an energetic unbalance [Wallace \& Klein, 1969, Ueba et al, 1973].

We have used ISO-induced MI, which is a non-invasive experimental model, to study the mechanisms involved in MI in the steps previous and posterior to infarction [Chagoya de Sánchez et al., 1997]. Myocardial lesions induced by ISO are related to its cardiac stimulating properties [Chappel et al.,1959]. Our group has characterized histological, physiological and biochemical alterations in a long term model (0-96h) of ISO administration to rats [Chagoya de Sánchez et al., 1997]. Animals treated with ISO showed an infarct-like damage of the circumferential type in the subendocardium at the apex region of the left ventricle that

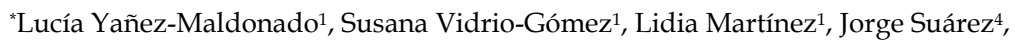
Alberto Aranda-Fraustro' ${ }^{2}$ Juan Carlos Torres ${ }^{3}$ and Gabriela Velasco-Loyden ${ }^{1}$ 2Departamento de Patología, Instituto Nacional de Cardiología, "Ignacio Chávez", Mexico 3Departamento de Farmacología, Instituto Nacional de Cardiología, "Ignacio Chávez", Mexico ${ }^{4}$ Current address: Department of Medicine, University of California, San Diego, CA, USA
} 
occurred at 12-24h after ISO administration. MI lesion was defined by: 1) histological criteria looking for coagulative necrosis and fiber fragmentation, 2) physiological and biochemical criteria, 3) functionally by a continuous telemetric ECG recordings and 4) the increase of serum marker enzymes specific for myocardial damage. These results lead us to define three stages of myocardial damage induced by ISO, preinfarction (0-12 h), infarction (12-24 h) and postinfarction (24-96 h).

During the physiological characterization of the ISO model, the first functional events observed after 2 minutes of ISO administration were a 70\% increase in heart rate and a $40 \%$ and $30 \%$ decrease in diastolic and systolic blood pressure respectively, which lasted from 3 minutes to $6 \mathrm{~h}$ after ISO administration, inducing a functional hypoxia that resulted in a MI. Ultra structural changes in mitochondria were evident from the first hour of treatment but functional alterations in isolated mitochondria, like oxygen consumption, respiratory quotient, ATP synthesis and membrane potential appeared at $6 \mathrm{~h}$ of drug administration. However, an important decrease in mitochondrial protein (50\%) was noticed after $3 \mathrm{~h}$ of treatment and was maintained during the whole study, but the energy imbalance, reflected by a decrease in energy charge and in the creatine phosphate/creatine ratio, was observed after 30 minutes of the treatment. All these alterations reached a maximum at the onset of infarction. Partial recovery of some of these parameters was observed during post-infarction period (24-96h) but it was not the case for ATP synthesis, oxygen consumption, total adenine nucleotides, and mitochondrial protein [Chagoya de Sánchez et al., 1997]. These results showed the critical role of mitochondrial function in the energy unbalance at the onset of MI induced by ISO.

Early changes in $\mathrm{Ca}^{2+}$ overload and their consequences are crucial in ISO cardiotoxicity [Fleckenstein et al.,1974, Bloom \& Davis, 1972; Barry \& Bridge, 1972]. Prolonged hypoxia and the inotropic action of ISO on the cardiac muscle make it very likely that oxidative stress and $\mathrm{Ca}^{2+}$ overload could be involved in the mechanism of ISO-induced MI. Deregulation of intracellular $\mathrm{Ca}^{2+}$ homeostasis modifies the cell redox state generating oxidative stress [Choudhary \& Dudley, 2002]. Knowing that myocardial $\mathrm{Ca}^{2+}$ overload and oxidative stress are well documented effects of ISO-induced MI, we investigated the correlation between oxidative stress and intracellular calcium handling in our model [DíazMuñoz et al., 2006]. Analysis of total calcium content in sub cellular fractions, revealed that the mitochondrial fraction presented a significantly elevated $\mathrm{Ca}^{2+}$ content $(80 \%)$ after 30 minutes of ISO treatment reaching a peak at $6 \mathrm{~h}(220 \%)$ and returning towards normal values after the MI (24-48h). These results showed a good correlation with the energy unbalance previously commented.

The oxidative stress in the ISO-induced myocardial infarction was evaluated studying lipid peroxidation, the free radical generating systems, antioxidant enzymes and the glutathione system [Díaz-Muñoz et al., 2006]. Oxyradical generation during myocardial ischemia occurs mainly through the xanthine oxidase and the activated neutrophils [Ray \& MaCord, 1982; Reimer et al., 1989] In addition, ISO oxidation also generates superoxide anions [Yates et al., 1981]. In our model, a large amount of superoxide anions are formed during the preinfarction phase by inhibition of superoxide dismutase and catalase, just after MI there is an increase in the activity of both enzymes inactivating the superoxide ion. The nitroradicals 
during MI are related with nitric oxide formation quantified by the stable products nitrite and nitrates ions. Lipid peroxidation was clearly increased after 6-96 h of ISO treatment as a consequence of ion superoxide formation during the pre-infarction period And the nitric oxide (NO) generated throughout the experimental period. The glutathione cycle, studied by the content of GSH and GSSG and the activities of GSH-peroxidase and GSH-reductase, was decreased during the three stages of ISO-induced cardiotoxicity. Thus, confirming the importance of the oxidative stress generated by an increase in ROS and RNS as well as a decrease in antioxidant defenses in this experimental model, reaching a maximum at 24-48 $\mathrm{h}$ [Díaz-Muñoz et al., 2006].

Niric oxide is a small biological molecule generated as gaseous free radical that participates in multiple physiological and pathological processes. It is a very reactive molecule with a life span of a few seconds that diffuses freely across membranes. To date, NO is considered as an important messenger molecule. In 1987 Moncada's group [Palmer et al., 1987] showed that the endothelium derived relaxing factor (EDRF) was the nitric oxide that is biosynthesized from L-arginine [Palmer et al., 1988]. Later on, it was shown that NO formation is not limited to vascular tissue but into numerous cells. Apparently, it is a general mechanism of intercellular communication in order to activate the guanylate cyclase [Berrazueta et al., 1990]. NO is also a main regulator of the immune activity and functions as neurotransmitter in the central nervous tissue as physiological messenger [Lowestein \& Snyder,1992] but it also has an important cytotoxic activity in vivo [Kröncke et al., 1997]. Physiological NO is synthesized by the constitutive nitric oxide synthase (eNOS, or III) for short periods of time (seconds or minutes). Cytotoxic NO is synthetized by the inducible nitric oxide synthase (iNOS or II) which produces NO for large periods of time, hours or days [Laurent et al., 1996] as occurs in macrophages activation.

NO action can be direct or indirect [Espey et al., 2000; Wink \& Mitchell, 1998]. In the direct one, NO interacts rapidly with the biological blank as occurs in the activation of the guanylate cyclase. In this case, NO reacts directly with the iron of the hemo group of the active center of the enzyme generating GMPc, which activates a cGMP dependent protein kinase resulting in the relaxation of the smooth muscle and vasodilatation, diminished heart contraction and stimulation of ionic pumps [Ignarro, 1990; Kröncke, 1997]. On the other hand, indirect effects are related to oxygen and nitrogen reactive species generating peroxynitrites (ONOO-). These compounds are responsible for the cytotoxicity that induces DNA fragmentation or lipid oxidation. Peroxynitrtites induce damage of the I-IV segments of the respiratory chain in the mitochondria as well as damage of Mn dependent superoxide dismutase (SOD-Mn) increasing the generation of $\mathrm{H}_{2} \mathrm{O}_{2}$ and superoxide anion which results in a dysfunctional mitochondria [Brown, 1999]. NO in the extracellular medium will react with oxygen and water to form nitrites and nitrates.

Nitric oxide is produced by three isoforms of nitric oxide synthases in the heart; endothelial (eNOS), inducible (iNOS) and neuronal (nNOS). Cardiac myocytes express the three isoforms although eNOS is mainly expressed in the endothelial cells. eNOS is constitutively expressed and can be stimulated with the increase of cytosolic calcium and phosphorylation [Cale \& Bird, 2006]. In contrast, iNOS is a cytokine-inducible isoenzyme. In healthy heart, physiological amounts of NO may help to sustain cardiac inotropy [Krenek et al., 2009]. It is 
our hypothesis that in the ISO-induced MI model, the hypotensive effect observed after ISO administration, in the preinfarct stage [Chagoya de Sánchez, 1997], is mediated by NO and might be critical for the MI development. The main goal of this study is to investigate the role of NO in the development of MI using an inhibitor of its synthesis L-NAME (nitro-Larginine methyl ester). We examined the relative role of eNOS and iNOS on IM-induced pathological changes of histological, physiological, biochemical and molecular parameters as well as energy state of heart. Preinfarction, infarction and postinfarction stages were analysed.

\section{Material and methods}

Enzymes, coenzymes, isoproterenol, L-NAME and the kits for the assessment of enzyme activities were from Sigma Chemical Co (St. Louis, MO). eNOS and iNOS antibodies were obtained from Santa Cruz Biotechnology (Santa Cruz, CA). All other reagents were obtained from Merck (Mexico).

\subsection{Animal treatment}

Male Wistar rats (250-300g, body weight) were injected subcutaneously (s.c.) at 9 AM with a single dose of isoproterenol hydrochloride $(67 \mathrm{mg} / \mathrm{kg}$ body weight). Control animals were injected with saline solution. Animals were euthanized at $0.5,1,3,6,12,24,48,72$, and $96 \mathrm{~h}$ after treatment, with exception of the animals used for hemodynamic parameters by radiotelemetry. Blood samples were taken to obtain serum. A heart sample was used for histological studies; other sample was taken for perchloric extracts and for analysis of the expression of the nitric oxide synthases. Other set of experiments in similar conditions were performed for Langendorff preparations to evaluate NO formation. L-NAME was administered i.p. at the dose of $110 \mathrm{mg} / \mathrm{kg}$ of body weight one hour before the ISO treatments. All procedures were conducted in accordance to the Federal Regulation for Animal Care and Experimentation (Ministry of Agriculture, SAGAR, Mexico).

\subsection{Histological studies}

The heart was removed immediately after death and slices were cut from the apex, fixed with $10 \%$ neutral buffered formaline. After embedding tissue in paraffine $4 \mu \mathrm{m}$ thickness sections were stained with hematoxilin-eosine and Masson's trichrome. A qualitative evaluation was made with a light microscope considering elongation, ondulation of the fibers, formation of contractile bands as a characteristic of the pre infarction stage, and the presence o macrophages, fibroblasts and collagen fibers as a post infarction stage.

\subsection{Hemodynamic studies}

These parameters were studied by radiotelemetry in freely moving conscious animals with a Data Sciences International equipment (Brockway and Hassier 1993), using a TL11M2-C50PXT implant to monitor blood systolic and diastolic pressure, and heart rate. To implant transmitters, rats were anesthetized with an i.p. injection of ketamine plus xylazine at 80 and $10 \mathrm{mg} / \mathrm{kg}$ body weight respectively, surgical conditions as previously described [Chagoya 
de Sánchez et al., 1997]. Animals were allowed to recover at least for one week. Twenty four-hours recordings were taken as control of the parameters to be studied. Afterwards, ISO was s.c. administered. L-NAME was administered one hour before ISO administration in the corresponding groups.

\subsection{Biochemical studies}

Nitric oxide formation was measured in isolated perfused hearts as described previously [Suárez et al., 1999]. Briefly, animals were anesthetized with sodium pentobarbital $(63 \mathrm{mg} / \mathrm{kg}$ of body weight). The heart was removed and retrogradely perfused via a non-recirculating perfusion system at constant flow. Perfusion medium was Krebs-Henseleit solution equilibrated with $95 \% \mathrm{O}_{2}-5 \% \mathrm{CO}_{2}$ at $37 \circ \mathrm{C}$ and $\mathrm{pH}$ of 7.4 , with $2 \mu \mathrm{M}$ oxihemoglobin solution for $\mathrm{NO}$ further quantification. Once the heart had been connected, it was maintained 30 minutes (perfusion flow rate of $25 \mathrm{ml} / \mathrm{min}$ for 5 minutes, then the following 25 minutes at $10 \mathrm{ml} / \mathrm{min}$ ) and cardiac frequency was stabilized at $1 \mathrm{~Hz}$. Perfusion flow rate was controlled by a peristaltic pump and 3 samples spaced by 5 minutes were taken from the coronary arteries. NO was measured indirectly in a double bean spectrophotometer (DW2000, SLM Aminco, Urban Illinois USA) quantifying the metahemoglobin produced as a function of NO generated in the coronary arteries [Kelm \& Schrader, 1988]. NO serum levels were measured indirectly by the quantification of nitrites and nitrates ions with the Grieiss reagent [Shultz et al., 1991]. NO synthase activity was measured by monitoring the conversion of $\left[{ }^{3} \mathrm{H}\right]$-Arginine to $\left[{ }^{3} \mathrm{H}\right]-$ Citrulline using a NOS Activity Assay Kit (Cayman Chemical Co, Ann Arbor, MI) and [ $\left.{ }^{3} \mathrm{H}\right]-$ Arginine (PerkinElmer, Inc, Boston, MA) according to the manufacturer's instructions. Briefly, $40 \mu \mathrm{g}$ of heart homogenate were used for the assay, and the reaction was carried out for $30 \mathrm{~min}$ at $37^{\circ} \mathrm{C}$. Serum levels of myocardial-damage enzyme markers were measured from blood samples taken from the neck of the rat at the time of euthanasia. Creatine phosphokinase (CK) (EC2.7.3.2) and its heart isoenzyme (CK-MB), as well as alpha-hydroxybutyrate dehydrogenase (alpha-HBDH, EC 1.1.1.30) were determined using conventional diagnostic kits. Aspartate aminotransferase (AST, EC 2.6.1.1) and lactic dehydrogenase (LDH, EC1,1,1,27) were measured by the method of Bergmeyer and Bernt [1965] and [Bergmeyer etal. 1965]. Energy metabolism in the heart was evaluated measuring adenine nucleotides ATP, ADP and AMP and energy parameters as total adenine nucleotides and energy charge. For adenine nucleotide determination, $300 \mathrm{mg}$ of the heart were extracted with $8 \%$ perchloric acid, after centrifugation the sample was neutralized with $4 \mathrm{M} \mathrm{K} \mathrm{K}_{2} \mathrm{CO}_{3}$ Adenine nucleotide were quantified by reversed-phase high performance liquid chromatography [Hoffman\& Liao, 1977]. Energy charge was calculated according to Atkinson [1968].

\subsection{Protein expression by western blotting}

Apical part of the left ventricle were homogenized in $50 \mathrm{mM}$ Tris, $\mathrm{pH} 7.4,1 \mathrm{mM}$ EDTA, containing a mixture of protease inhibitors. Homogenates were centrifuged at 10,000 $\mathrm{g}$ for 15 $\mathrm{min}$, and the supernatant was used as whole cell protein. Protein samples $(20 \mu \mathrm{g}$ of protein/well) were electrophoresed in SDS-polyacrylamide gels and transferred to PDVF membranes. Membranes were blocked for $1 \mathrm{~h}$ with 5\% nonfat dry milk in TBST (50 mM Tris, $150 \mathrm{mM} \mathrm{NaCl}$ at $\mathrm{pH} 7.4$, and $0.05 \%$ Tween 20). Proteins were detected by incubating membranes overnight at $4^{\circ} \mathrm{C}$ with the following primary antibodies: anti-eNOS and anti-iNOS from Santa Cruz Biotechnology (Santa Cruz, CA), anti-phospho-eNOS (Ser-1177) (Cell 
Signaling Technology, Danvers, MA). Primary antibody binding was detected with the respective horseradish peroxidase-conjugated secondary antibody (Santa Cruz Biotechnology, Santa Cruz, CA). Densitometric analysis of bands was performed with the Quantity One software (Bio-Rad).

\section{Results}

\subsection{Histological studies}

Histological evaluation of the myocardial lesion induced by isoproterenol treatment with and without L-NAME is shown on Table 1. The ISO-induced lesion is localized in the apical region of the left ventricle. In the presence of L-NAME, ISO-induced lesion maintained the same localization but it was extended to the subepicardial zone with less intensity. The preinfarct lesions; elongation and ondulation of the fibers and necrosis and contraction bands were prolonged for $24 \mathrm{~h}$. The infarct lesions persisted for $48 \mathrm{~h}$ and the post infarction initiated early at $24 \mathrm{~h}$ with a slight decrease. Although the damaged fibers were similar in both groups, no necrosis was observed at the pre infarction stage suggesting that acute phase of necrosis at $6 \mathrm{~h}$ observed in the ISO group decreased and was extended for a longer period in presence of L-NAME. A diminished presence of polymorphonuclear cells and macrophages indicates a minor cellular lesion in the group treated with L-NAME demonstrating a different histological profile for each group.

\begin{tabular}{|c|c|c|c|c|c|c|c|c|c|c|c|c|c|c|}
\hline \multirow[b]{2}{*}{ Type of damage } & \multicolumn{5}{|c|}{ Time of ISO treatment (hours) } & \multicolumn{7}{|c|}{ Time of ISO-L-NAME treatment (hours) } & \multirow[b]{2}{*}{72} & \multirow[b]{2}{*}{96} \\
\hline & 3 & 6 & 12 & 24 & 48 & 72 & 96 & 3 & 6 & 12 & 24 & 48 & & \\
\hline $\begin{array}{l}\text { Elongation and } \\
\text { ondulation of fibers }\end{array}$ & +++ & ++ & + & - & - & + & + & ++ & ++ & +++ & ++ & + & - & - \\
\hline $\begin{array}{l}\text { Necrosis and } \\
\text { contraction bands }\end{array}$ & + & +++ & ++ & + & - & - & - & ++ & + & ++ & +++ & + & + & ++ \\
\hline Coagulative necrosis & - & - & + & ++ & - & - & - & - & $+/-$ & + & ++ & + & - & - \\
\hline $\begin{array}{l}\text { Fragmentation of } \\
\text { myofibrils }\end{array}$ & - & - & - & + & ++ & - & - & - & - & $+/-$ & + & ++ & ++ & - \\
\hline $\begin{array}{l}\text { Polymorphonuclear } \\
\text { cells }\end{array}$ & - & + & ++ & +++ & + & - & - & - & - & + & ++ & + & - & - \\
\hline Macrophages & - & - & - & + & +++ & ++ & + & - & - & - & + & ++ & ++ & + \\
\hline $\begin{array}{l}\text { Fibroblasts and } \\
\text { collagen fibers }\end{array}$ & - & - & - & - & + & ++ & +++ & - & - & - & + & ++ & +++ & ++ \\
\hline Edema & - & + & ++ & +++ & ++ & + & - & + & + & +++ & ++ & + & - & $+/-$ \\
\hline
\end{tabular}

Table 1. Histological evaluation of the myocardial lesion by isoproterenol (ISO) with and without L-NAME. Animals received an s.c. administration of ISO. L-NAME was administrated 1 hour before. They were euthanized after ISO administration. Total damage with ISO was taken as $100 \%$. Score code is: $(+)=25 \% ;(++)=50 \% ;(+++)=75 \% ;(-)=$ not observed.

\subsection{Physiological studies}

Blood pressure and heart rate were monitored continuously by telemetry, the results are shown in the Figure 1. To study the effect of L-NAME it was administered i.p. $1 \mathrm{~h}$ before ISO, its effect was detected in the pre-ISO lecture, the initial ISO effect was observed at time 0. A) Heart rate shows a strong and immediate increase from 279 to 450 b.p.m. during the first minutes of ISO administration. A further small increase $(20 \%)$ was observed in the 
group of animals treated with L-NAME until 48h of treatment. B) Changes in systolic blood pressure show the hypotensive effect of ISO previously described (Chagoya de Sánchez et al., 1997). The group treated with L-NAME showed an increase in systolic pressure from 85 to $155 \mathrm{mmHg}$. ISO administration induced a diminution towards normal values followed by an increase to $140 \mathrm{mmHg}$ at $3 \mathrm{~h}$ of treatment returning to control values at $24 \mathrm{~h}$. C) Similar profile was observed with diastolic pressure but the hypertensive effect $(180 \mathrm{mmHg}$ ) was more evident. L-NAME administration did not significantly affect the effect of ISO increasing the heart rate but significantly reduced the hypotensive effect of ISO.

B
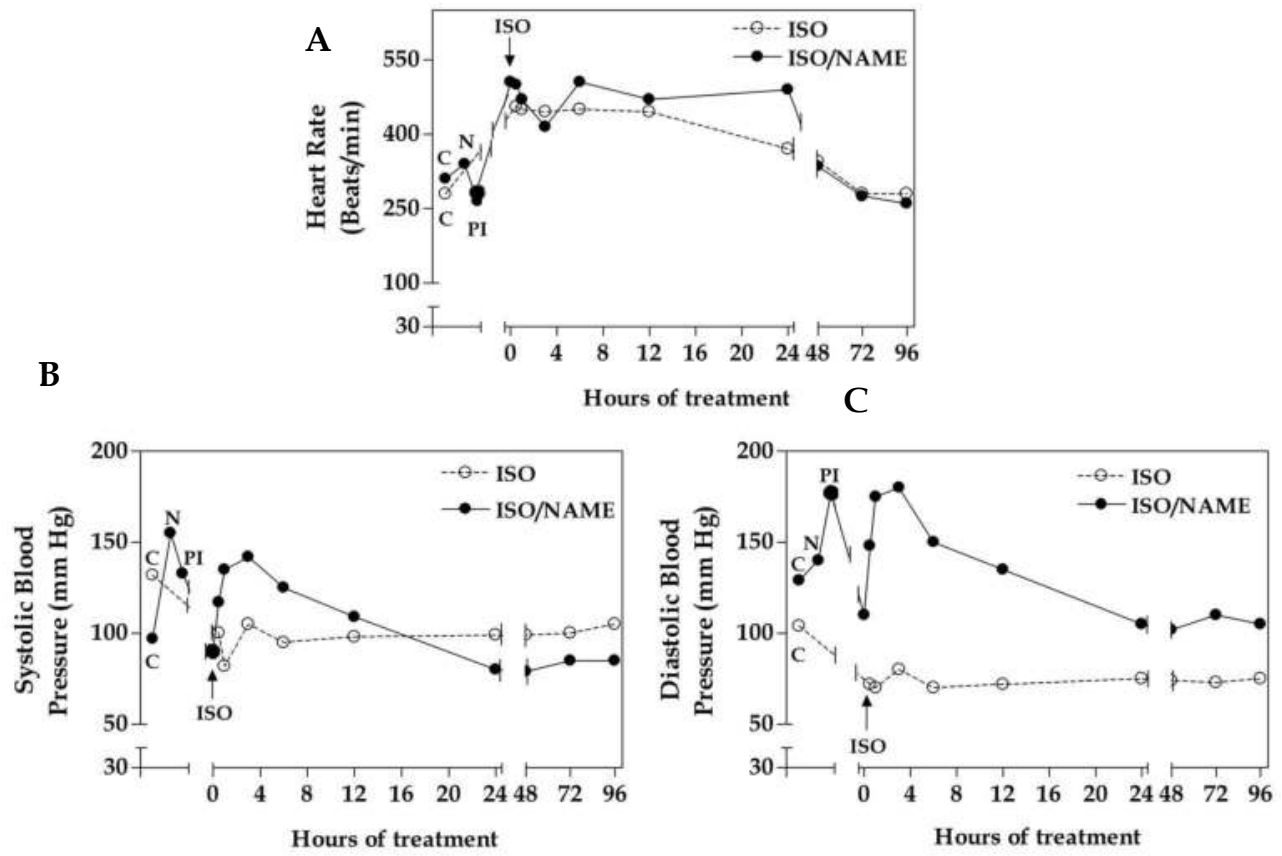

Fig. 1. In vivo effect of L-NAME on changes induced by isoproterenol on heart rate and blood pressure. Rats received (i.p) NAME one hour before isoproterenol injection (s.c) at time 0 , as described in Material and methods. The response was monitored continuously by a telemetry system. $\mathrm{C}=$ Control, $\mathrm{N}=\mathrm{NAME}$ and $\mathrm{PI}=$ Pre-ISO. A) Time course of the variation in heart rate (b.p.m.; beats per minute) B) Systolic pressure (mmHg) and C) Diastolic pressure $(\mathrm{mmHg})$. Values are from a representative experiment of five.

\subsection{Biochemical studies}

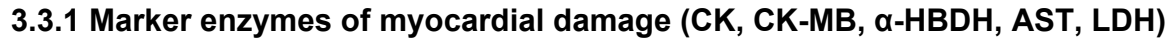

Were measured in serum of the experimental animals at the time of infarction that is at $12 \mathrm{~h}$ after ISO treatment in the presence or absence of L-NAME. Results are presented in the Table 2. ISO-induced cardiac lesions caused a 2 to 10-fold increase in marker enzymes including specific enzymes of cardiac origin like CK-MB which increased almost 4-fold with respect to its control values. Enzymes representative of cell necrosis, as LDH, showed a 
tenfold augmentation. Animals treated with L-NAME and ISO showed a significant reduction in the level of marker enzymes mainly CK-MB and $\mathrm{LDH}$ indicating diminished myocardial damage at that time in the group of animals treated with L-NAME.

\begin{tabular}{llcc}
\hline Enzymes & $\begin{array}{l}\text { Without } \\
\text { treatment }\end{array}$ & ISO & ISO/NAME \\
\hline CK & $110 \pm 9.2$ & $\begin{array}{c}220 \pm 23 \\
\mathrm{P}<0.005\end{array}$ & $\begin{array}{c}80 \pm 2.4 \\
\mathrm{P}<0.001\end{array}$ \\
\hline \multirow{2}{*}{ CK MB } & \multirow{2}{*}{$104 \pm 6.5$} & $\begin{array}{c}389 \pm 50.0 \\
\mathrm{P}<0.001\end{array}$ & $\begin{array}{c}160 \pm 3.5 \\
\mathrm{P}<0.005\end{array}$ \\
\hline \multirow{2}{*}{$\alpha-\mathrm{HBDH}$} & $22 \pm 1.9$ & $50 \pm 8.0$ & $\begin{array}{c}44 \pm 3.9 \\
\mathrm{P}<0.01\end{array}$ \\
\hline \multirow{2}{*}{ AST } & \multirow{2}{*}{$110 \pm 7.0$} & $\begin{array}{c}260 \pm 16.8 \\
\mathrm{P}<0.001\end{array}$ & $\begin{array}{c}220 \pm 10.0 \\
\mathrm{P}<0.1\end{array}$ \\
\hline \multirow{2}{*}{ LDH } & \multirow{2}{*}{$120 \pm 5.6$} & $\begin{array}{c}1180 \pm 13.0 \\
\mathrm{P}<0.001\end{array}$ & $\begin{array}{c}102 \pm 2.0 \\
\mathrm{P}<0.001\end{array}$ \\
\hline
\end{tabular}

Table 2. Effect of isoproterenol and L-NAME on serum levels of marker enzymes of myocardial damage at the time of infarction $(12 \mathrm{~h})$. Values are average activity of the different enzymes in serum samples of the rats $(n=5) \pm$ standard error. $P$ value of the isoproterenol column represents a comparison with the control group without treatment and the P value of the ISO-NAME group is the significance of the L-NAME/ISO group vs. ISO group.

\subsubsection{Energy metabolism}

The influence of L-NAME on the changes in energy metabolism produced by isoproterenol (Chagoya de Sánchez et al., 1997) is shown in Table 3. Values at time 0 were obtained in animals without treatment. Values of the Pre-ISO group correspond to the group that received L-NAME treatment one hour before ISO administration. The energy unbalance generated by ISO treatment is clearly observed by the statistically significant decrease in ATP levels along the three stages of cardiotoxicity; pre infarction, infarction and post infarction, being more decreased at $24 \mathrm{~h}$ during the infarction period. These changes are also accompanied by a diminution of ADP and an increase in AMP levels resulting in low levels of total adenine nucleotides in the heart with decreased energy charge $($ E.C. $=[$ ATP $]+1 / 2$ $[\mathrm{ADP}] /[\mathrm{ATP}]+[\mathrm{ADP}]+[\mathrm{AMP}](\mathrm{AN}=$ total adenine nucleotides$)$, being 0.86 a normal value). Animals treated with L-NAME also presented an energy unbalance ATP and ADP levels were higher than those observed in the ISO group. AMP levels increased only at 3 and $6 \mathrm{~h}$ and an interesting observation is that during the pos infarction stage energy parameters (ATP, total adenine nucleotides and energy charge) recovered towards normal values.

\subsubsection{NO formation}

We evaluated NO release in isolated perfused hearts from experimental animals at different times of ISO treatment (Fig 2). An increase in NO release was observed since $0.5 \mathrm{~h}$ reaching a maximum at 6 and $12 \mathrm{~h}$ of ISO treatment. NO release levels returned to control values from 24 to $96 \mathrm{~h}$. L-NAME inhibitory effect on ISO-induced NO release was evident since 


\begin{tabular}{ccccccccccc}
\hline $\begin{array}{c}\text { Time of } \\
\text { Treatment }\end{array}$ & \multicolumn{2}{c}{ ATP } & \multicolumn{2}{c}{ ADP } & \multicolumn{2}{c}{ AMP } & & AN & EC \\
\hline Hours & ISO & ISO/NAME & ISO & ISO/NAME & ISO & ISO/NAME & ISO & ISO/NAME & ISO & ISO/NAME \\
\hline Pre-ISO & - & $4.9 \pm .40$ & - & $2.5 \pm .22$ & - & $1.3 \pm .20$ & - & $7.99 \pm .33$ & - & $0.71 \pm .01$ \\
\hline $\mathbf{0}$ & $6.2 \pm .50$ & - & $2.7 \pm .10$ & - & $0.4 \pm .08$ & - & $9.1 \pm .59$ & - & $0.8 \pm .01$ \\
\hline $\mathbf{3}$ & $3.0 \pm .20^{*}$ & $3.9 \pm .60$ & $3.1 \pm .40$ & $2.8 \pm .66$ & $1.4 \pm .20^{*}$ & $2.1 \pm .16^{* \#}$ & $7.3 \pm .73^{*}$ & $8.0 \pm .99$ & $0.6 \pm .01^{*}$ & $0.6 \pm .008^{*}$ \\
\hline $\mathbf{6}$ & $2.7 \pm .20^{*}$ & $2.6 \pm .10^{*}$ & $2.0 \pm .05^{*}$ & $2.2 \pm .11$ & $1.5 \pm .12^{*}$ & $1.5 \pm .08$ & $5.6 \pm .17^{*}$ & $5.7 \pm .16^{*}$ & $0.61 \pm .02^{*}$ & $0.61 \pm .01^{*}$ \\
\hline $\mathbf{1 2}$ & $2.6 \pm .18^{*}$ & $2.6 \pm .20^{*}$ & $1.8 \pm .27^{*}$ & $1.9 \pm .05^{* \#}$ & $1.4 \pm .37^{*}$ & $1.2 \pm .29$ & $5.6 \pm .49^{*}$ & $6.0 \pm .66^{*}$ & $0.61 \pm .05^{*}$ & $0.57 \pm .02^{* \#}$ \\
\hline $\mathbf{2 4}$ & $1.5 \pm .10^{*}$ & $2.7 \pm .05^{* \#}$ & $1.0 \pm .08^{*}$ & $1.5 \pm .03^{* \#}$ & $0.9 \pm .15^{*}$ & $1.1 \pm .08$ & $3.3 \pm .09^{*}$ & $4.8 \pm .03^{* \#}$ & $0.6 \pm .02^{*}$ & $0.67 \pm .01^{* \#}$ \\
\hline $\mathbf{4 8}$ & $3.4 \pm .10^{*}$ & $3.9 \pm .60$ & $1.3 \pm .05^{*}$ & $2.0 \pm .16^{* \#}$ & $0.4 \pm .04^{*}$ & $1.1 \pm .04^{\#}$ & $5.0 \pm .09^{*}$ & $6.5 \pm .66^{\#}$ & $0.77 \pm .005^{*}$ & $0.71 \pm .02^{* \#}$ \\
\hline $\mathbf{7 2}$ & $3.2 \pm .05^{*}$ & $6.4 \pm .40^{* \#}$ & $1.9 \pm .10^{*}$ & $3.0 \pm .16^{* \#}$ & $1.1 \pm .07^{*}$ & $1.2 \pm .01$ & $4.3 \pm .16^{*}$ & $9.6 \pm .49^{* \#}$ & $0.67 \pm .005^{*}$ & $0.81 \pm .01^{* \#}$ \\
\hline $\mathbf{9 6}$ & $2.3 \pm .10^{*}$ & $4.0 \pm .20^{* \#}$ & $1.5 \pm .16^{*}$ & $2.3 \pm .27^{\#}$ & $0.4 \pm .04$ & $1.5 \pm .33^{\#}$ & $4.2 \pm .33^{*}$ & $7.3 \pm .33^{\#}$ & $0.72 \pm .03^{*}$ & $0.70 \pm .04^{*}$ \\
\hline
\end{tabular}

Table 3. Effect of L-NAME on changes of rat heart adenine nucleotides in the ISO-induced infarction. AN (adenine nucleotides) is de sum of adenine nucleotides; EC, energy charge $(\mathrm{ATP}+1 / 2 \mathrm{ADP} / \mathrm{AN})$ values are the means $\pm \mathrm{SEM}, \mathrm{n}=4,{ }^{*} \mathrm{p}<0.05$ ISO vs. 0 or ISO/NAME vs. Pre-ISO, \#p<0.05 ISO-NAME vs. ISO.

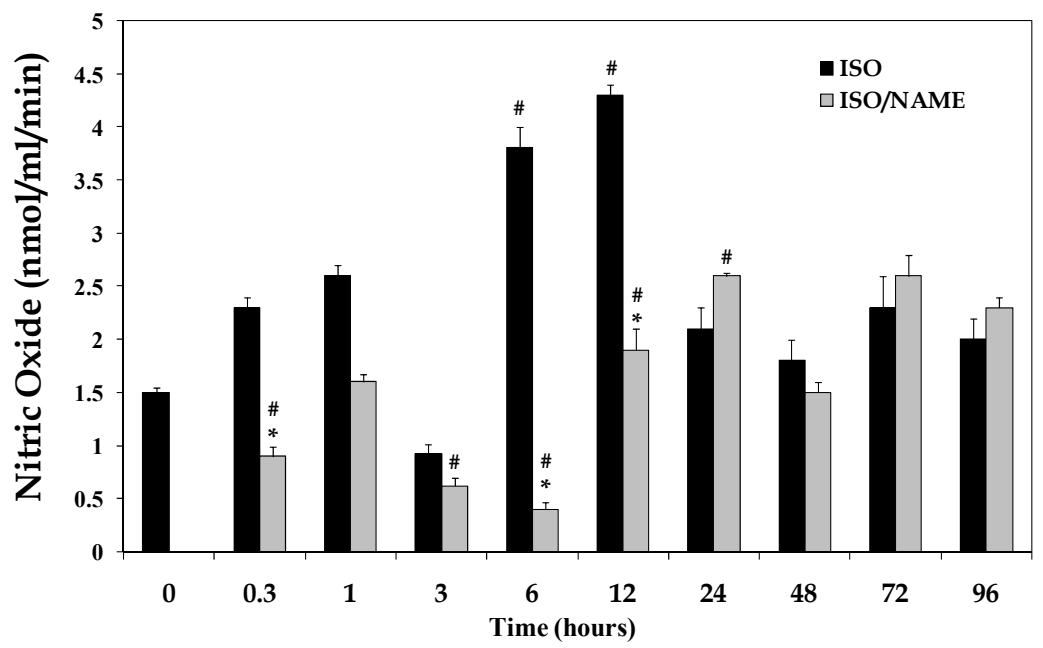

Fig. 2. Effects of isoproterenol and L-NAME on NO release. NO release in perfused hearts from treated rats at different times. Values are mean \pm S.E $n=5$. Dark bars are isoproterenol treatment and gray bars L-NAME + isoproterenol.\# $\mathrm{p} \leq 0.05$ ISO or ISO/NAME group vs Control (Time 0$) .{ }^{*} \mathrm{p} \leq 0.05$ ISO/NAME group vs ISO.

$0.5 \mathrm{~h}$ with a maximal effect at 6-12 $\mathrm{h}$ of ISO treatment. After this time, the amount of NO released was similar in both groups suggesting the end of the inhibitory effect of L-NAME. Fig 3, shows nitric oxide synthases activity of the constitutive form (eNOS) and the inducible form (iNOS). ISO treatment gradually increased the activity of both isoforms during the pre infarction and infarction period. The maximal increase was at $24 \mathrm{~h}$ for eNOS and $12 \mathrm{~h}$ for iNOS. After this time, during the postinfarction, there was an important 
decrease on both isoforms. Animals treated with L-.NAME showed a gradual diminution of eNOS activity $(3,24 \mathrm{~h})$. After this time the activities of the isoforms reach normal values. The increase in nitric oxide release during the pre infarction periods are the result of an increase in the activity of eNOS and iNOS and L-NAME decreased NO release by decreasing eNOS activity during the preinfarction-infarction stages since no significant change was observed in iNOS activity.

Protein expression of constitutive and inducible NO synthases in the presence of ISO and ISO+ L-NAME are shown in Fig 4. Small changes in the eNOS protein expression were observed during the preinfarction stage and more important ones during the post infarction Decreased eNOS protein levels were evident at 3,12, and $24 \mathrm{~h}$ in the presence of L-NAME and no appreciable changes during the post infarction. eNOS is activated by phosphorylation in serine 1177. An increase in p-eNOS was observed after ISO treatment $(1,3,6 \mathrm{~h})$ with a diminution at $(12,24, \mathrm{~h})$. Treatment with L-NAME markedly decreased p-
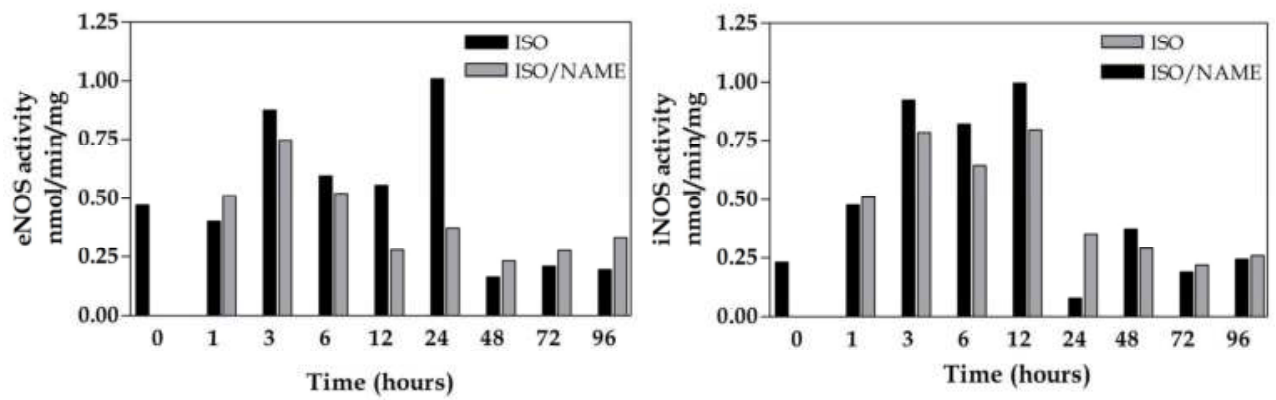

Fig. 3. The activity of constitutive and inducible (eNOS, iNOS) nitric oxide synthases. Values shown represent typical results of one experiment. Four experiments were performed.

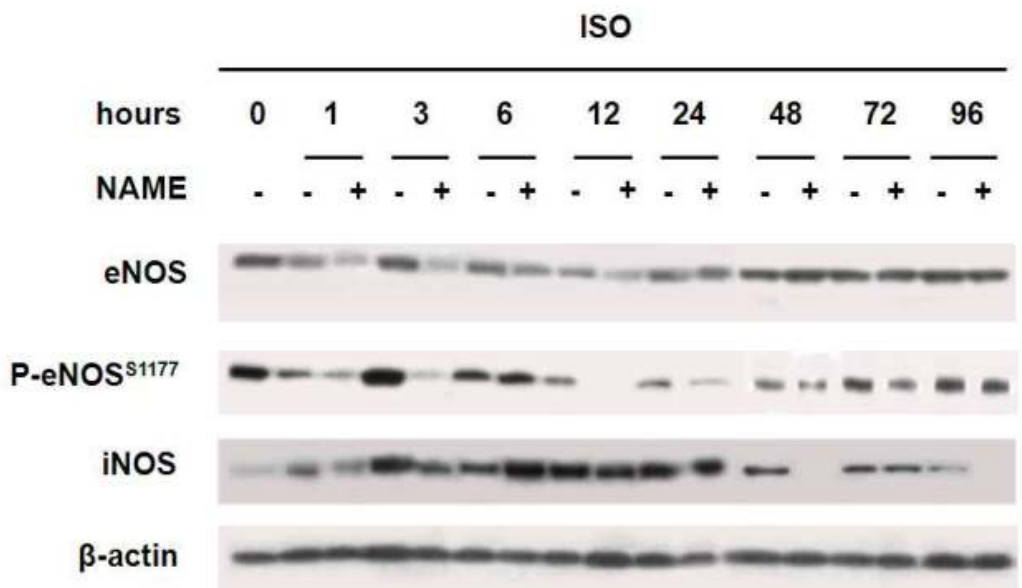

Fig. 4. Western blot analysis of a representative experiment (from three) of eNOS, P-eNOS s1177, iNOS protein expression. 
eNOs (S1177) at 3, 12, and $24 \mathrm{~h}$. At $6 \mathrm{~h}$ eNOS is phosphorylated and must be active, however, there is an important reduction in NO release in the presence of L-NAME. iNOS expression was not detected in the control group. However, after ISO treatment, there was a gradual increase until $12 \mathrm{~h}$ with an important decrease after infarction which reached almost the control value at $96 \mathrm{~h}$. A similar profile was observed in the group treated with L-NAME with differences at 6, 12, 48, and $96 \mathrm{~h}$. NO serum levels were measured indirectly quantifying nitrites and nitrates (Fig 5). Although the same tendency as in isolated perfused hearts was observed, increased NO release during infarction $(12,24 \mathrm{~h})$ was more clearly observed in the ISO-NAME group.

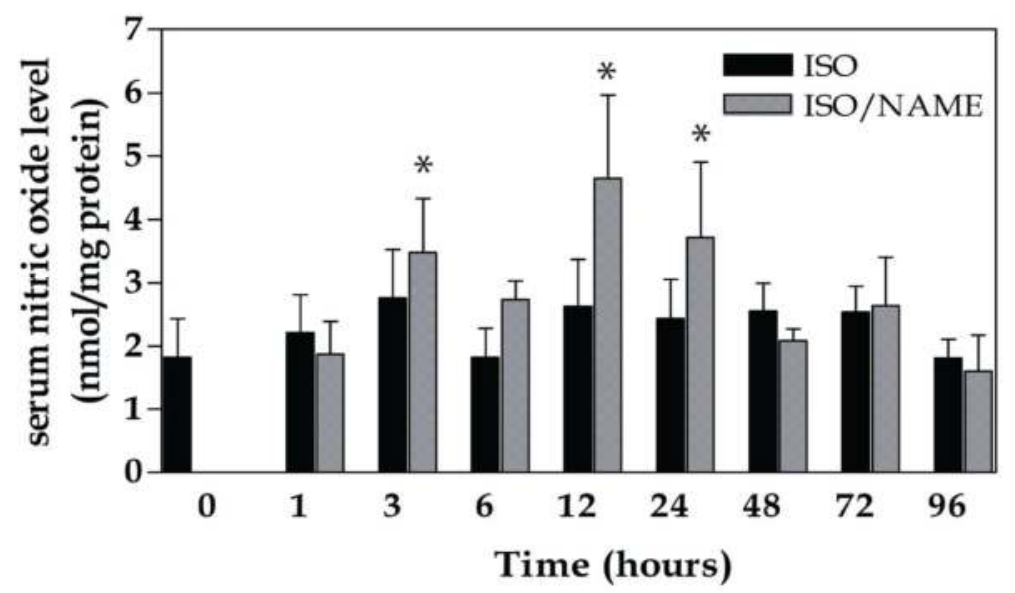

Fig. 5. $\mathrm{NO}_{2}+\mathrm{NO}_{3}$ in serum from both groups. Values are the mean $\pm \mathrm{SE}, \mathrm{n}=3$.

${ }^{*} \mathrm{p}$ values > 0.05 ISO/NAME group vs control (Time 0 )

\section{Discussion}

The role of nitric oxide in cardiovascular diseases is complex and controversial, mainly due to its multiple physiological and pathological effects which depend on the amount of NO synthetized by the nitric oxide synthases, their allosteric modulators, the metabolic conditions of the cells, redox and energy state, and the different experimental models used. A major physiological role of nitric oxide is the regulation of vascular tone. In this case the activity of endothelial eNOS is crucial since endothelial dysfunction is characterized by impairment of nitric oxide bioavailability which induces hypertension and cardiovascular diseases [Török, 2008]. In pathological conditions there is an increase in oxidative stress. Nitric oxide reacts with superoxide $\left(\mathrm{O}^{2-}\right)$ to produce peroxynitrites, highly cytotoxic nitrogen species, that induce DNA fragmentation, and damage of segments of the respiratory chain inducing dysfunctional mitochondria [Brown, 1999]. In the experimental model of myocardial infarction induced by isoproterenol studied here, we observed this dual actions of nitric oxide: 1) the marked diminution of systolic and diastolic pressure after 2 minutes of ISO treatment [Chagoya de Sanchez et al. 1997], suggesting the participation of the nitric 
oxide and 2) the cytotoxic action reflected in energy unbalance, dysfunctional mitochondria, oxidative stress, with increased levels of nitrites and nitrates as indicative of NO formation [Díaz-Muñoz et al, 2006).

In order to determine the role of nitric oxide in the ISO-induced MI, we used an inhibitor of endothelial nitric oxide synthase; L-NAME (NG-nitro-L-arginine methyl ester) [Sander et al. 1999; Frandsen et al. 2001] and systolic and diastolic blood pressure and heart rate were evaluated (Fig 1). The drastic decrease in systolic and diastolic blood pressure caused by isoproterenol could be related to an increase in nitric oxide release due to an overexpression of eNOS induced by isoproterenol as previously described [Krenek et al. 2006]. This idea was confirmed by our results that clearly showed a marked increase in NO release after ISO administration during the pre infarction stage $(0.5-12 \mathrm{~h})$. These results correlated with the increase in eNOS activity and protein expression of total eNOS. In addition, the activated form phosphorylated eNOS at Ser-1177 was increased during the pre infarction and infarction stages. During post-infarction phase, the expression of total and activated phosphorylated enzyme corresponds to a moderate increase in nitric oxide. This effect of isoproterenol is partially prevented by the presence of L-NAME and a decrease of 30 to $90 \%$ in NO release was observed (Fig2A). This reduction in NO release was also related to a decrease in eNOS activity and protein expression of total and phosphorylated (eNOS, PeNOSs1177. The eNOS-mediated NO generation is a highly regulated cellular event with multiple regulatory factors such as gene expression, translational modifications, intracellular localization, cofactors and phosphorylation [Govers \& Rabelink, 2001). This could be the case for the result obtained at $3 \mathrm{~h}$ of ISO administration in which NO release was low with elevated activity and expression of total and active form of eNOS. Isoproterenol also induced an increase of iNOS activity from 1-12 h of treatment reaching control values at the post infarction stage. Treatment with L-NAME induced a non-significant decrease in its activity and expression. The decrease in NO release and inhibition of eNOS in the presence of L-NAME lead us to the conclusion that the increase in systolic and diastolic pressure (Fig 1) contributes to improve heart function. The maintenance of the diastolic pressure is very important for a good perfusion of the heart through the coronary arteries. These effects were maintained throughout pre-infarction, infarction and post-infarction suggesting a decrease of cardiotoxicity induced by isoproterenol. This possibility was also tested measuring marker enzymes of myocardial damage in serum at the time of infarction (Table 1). The dramatic drop in CK-MB and LDH serum levels suggests that in the presence of L-NAME, the magnitude of myocardial damage was reduced. Previously it was shown that isoproterenol-induced MI is accompanied by a marked energetic unbalance through the 3 cardiotoxicity periods with no recovery in the post infarction stage. An interesting finding was that in the presence of L-NAME, at $48 \mathrm{~h}$ of ISO treatment in the post infarction stage, induced a recovery of energy balance which occurred increasing ATP, total adenine nucleotides and energy charge (Table 2). The indirect assessment of nitric oxide in serum also reflects the increase in NO at the infarction stage and it is mainly generated by iNOS when inflammatory cytokine levels are elevated as previously suggested [Gaballa et al., 1999]. Inhibition of eNOS by L-NAME blocks vessel dilation inducing a more extended lesion increasing the number of damaged cells. At same time, inflammatory cell migration diminishes by the vessel constriction induced by L-NAME resulting in less inflammation. 


\section{Conclusion}

In summary, the present study shows that NO plays an important role in the installation and progression of the myocardial infarction induced by isoproterenol. The increased generation of nitric oxide was evidenced from $0.5 \mathrm{~h}$ until $12 \mathrm{~h}$ of isoproterenol administration these likely resulted from an increase in the activity of both eNOS and iNOS. The first action seem to be a consequence of isoproterenol triggered signaling cascade action on the enzyme and the second one from subsequent inflammatory processes. The increase in eNOS is responsible for the hypotensive effect of ISO decreasing the systolic and diastolic pressure since the first 2 minutes of its administration. The consequent hypoperfusion induced an energy unbalance possibly due to the chronotropic and inotropic effects of isoproterenol which generates an increase in energy consumption. These effects demand an increase in mitochondrial function generating oxidative stress with ion superoxide formation, which in the presence of $\mathrm{NO}$, will generate peroxynitrites resulting in mitochondrial damage and cellular death. All above contribute to myocardial infarction and the dysfunctional mitochondria prevents recovery of energy balance at the post infarction stage. Experiments in the presence of L-NAME (NO inhibitor) clearly showed a diminution of the acute phase of infarct, the inflammatory process and the reduction of the hypotensive action of ISO. As a result, there was an important increase in diastolic pressure and perfusion of the heart through the coronary arteries, diminishing the oxidative stress and a recuperation of energy balance which contributed to ameliorate myocardial function. The infarction observed in the presence of L-NAME is likely involved by the inflammation process that is not affected significantly by L-NAME treatment. These results clearly show the action of NO in isoproterenol-induced myocardial infarction and suggest the importance to assess the role of $\mathrm{NO}$ in pre infarction and early post infarction stages in patients. It is possible that the therapeutic modulation of NO release might prevent the cytotoxic actions of the excessive NO release which facilitate the recovery of the patient at the post infarction stage.

\section{Clinical perspective}

Myocardial ischemia/reperfusion is the major threat to cardiac myocytes in acute myocardial infarction. In spite of early interventions like reperfusion therapy, myocardial damage can not be avoided. Consequently, additional options to enhance myocardial resistance to ischemia/reperfusion are needed. Increasing nitric oxide action has been considered a possibility to enhance myocardial perfusion during myocardial infarction. However, nitric oxide action may lead to adverse effects. We now add data on the beneficial effect of inhibiting nitric oxide action by using L-NAME during development of myocardial infarction. Inhibition of nitric oxide release diminished the inflammatory process and reduced the hypotensive action of isoproterenol. As a result, there was an important increase in diastolic pressure and perfusion of the heart. These effects diminished the oxidative stress and helped to improve energy balance which contributed to better recovery of the myocardial function. Hence, increasing nitric oxide action as a therapeutic regimen in acute myocardial infarction is likely to be less successful if the distinct phases of myocardial infarction development are not considered. Undoubtedly, nitric oxide-modulation of coronary vessel tone is a therapeutic target in myocardial infarction. In the present work, we demonstrated that inhibition of nitric oxide action can be beneficial depending of the 
infarction stage. Development of new therapeutic strategies focused to manipulate nitric oxide action in pre-infarction, infarction and post-infarction is necessary to alleviate this important cardiac disease.

\section{Acknowledgment}

This study was partially supported by grants from Dirección de Asuntos del Personal Académico (DGAPA IN-296589) and Consejo Nacional de Ciencia y Tecnología (M91090710). The authors acknowledge critical comments from Dr. Adolfo García-Sáinz as well as the assistance of M.C. Rebeca Pérez Cabeza de Vaca, and secretarial assistance of Miss Rosario Villaseñor.

\section{References}

Atkinson D.E. (1968) The energy charge of the adenylate pool as a regulatory parameter. Interaction with feedback modifiers. Biochemistry 7: 4030-4034, 1968.

Bergmeyer H.U. \& Bernt E. (1965) Glutamate-oxaloacetate transaminase. Colorimetric determination with with 2,4 -dinitrophenyl-hydrazine. In Methods of enzymatic analysis, p 837 Edited by H.U: Bergmeyer. Academic Press, New York.

Bergmeyer, HU., Bernt, E. \& Hess, B. (1965) Lactic dehydrogenase. In Methods of enzymatic analysis, p 736. Edited by H.U. Bergmeyer. Academic Press, New York.

Berrazueta J.R., López Jaramillo P., Moncada S. (1990) EL óxido nítrico: de vasodilatador endógeno a mediador endógeno. Revista Española de Cardiología 43: 421-431.

Brown G.C. (1999) Nitric oxide and mitochondrial respiration. Biochim. Biophys. Acta 1411: 351-369.

Cale J.M. \& Bird I.M. (2006) Dissociation of endothelial nitric oxide synthase phosphorylation and activity in uterine artery endothelial cell. Am J Physiol Heart Circ Physiol 290: H1433-H1445.

Chagoya de Sánchez V., Hernández-Muñoz R., López-Barrera F., Yañez L., Vidrio S., Suárez J., Cota-Garza M.D., Aranda-Frausro A., Cruz D. (1997) Sequential changes of energy metabolism and mitochondrial function in myocardial infarction induced by isoproterenol in rats: a long-term and integrative study. Can. J. Physiol. Pharmacol 75: 1300-1311.

Diaz-Muñoz M., Älvarez-Perez M.A., Yañez L., Vidrio S., Martínez L., Rosas G., Yañez M., Ramírez S., Chagoya de Sánchez V. (2006) Correlation between oxidative stress and alteration of intracellular calcium handling in isoproterenol-induced myocardial infarction. Mol Cell Biochem 289: 125-136.

Chappel C.I., Rona G., Balzs T. (1959) Comparison of cardiotoxic action.of certain sympathomimetic amines. Can J Blochem Physiol 37:35-42.

Espey M.G., Miranda K.M., Feelisch M., Fukuto J., Grisham M.B., Vitek M.P. and Wink D.A. (2000) Mechanism of cell death governed by the balance between nitrosative and oxidation stress. Ann. NY Acad. Sci. 899: 209-221.

Frandesen U, Bangsbo J, Sander M, Hoffner L, Betak A, Saltin B \& Hellsten Y (2001) Excercise-induced hyperaemia and leg oxygen uptake are not altered during 
effective inhibition of nitric oxide synthase with $\mathrm{NG}^{\mathrm{G}}$-nitro-L-arginine methyl ester in humans. J. Physiol 531:257-264.

Govers, R. \& T.J: Rabelink (2001) Cellular regulation of endothelial nitric oxide synthase. Am J Physiol, Renal Physiol 280: F193-F206.

Hoffman N.E., \& Liao J.C. (1977) Reversed phase high performance liquid chromatography separations of nucleotides in the presence of solvophobic ions. Anal. Chem 49:22313-2234.

Ignarro L.J. (1990) Biosynthesis and metabolism of endothelium-derived nitric oxide. Annu, Rev Pharmacol. Toxicol 30: 535-560.

Kelm M., Schrader J. (1988) Nitric oxide release from the isolated guinea heart. Eur. J. Pharmacol. 155: 317-321.

Krenek P, Kimas J. Krolakova M, Gasova A, Plandorova J, Kucerova D, Fecenkova A. Svec P, Kyselovic J. (2006) Increased expression of endothelial nitric oxide synthase and caveolin-1 in the aorta of rats with isoproterenol-induced cardiac hypertrophy .Can J Physiol Pharmacol 84: 1245-1250.

Krenek P., Kmecova J., Kucerova D., Bajuszova Z., Musil P., Gazova A., Ochodnicky P., Klimas J. \& Kyselovic J. (2009) Isoproterenol-induced heart failure in the rat is associated with nitric oxide-dependent functional alterations of cardiac function. European J Heart Failure 11: 140-146

Kröncke Klaus-D., Fehsel K. and Kolb Bachofen V. (1997) Nitric Oxide: Cytotoxicity versus Cytoprotection. How, Why, and Where? Nitric Oxide: Biology and Chemistry 1: 107-120. (Ignarro L.J. Annu. Rev Pharmacol. Toxicol 30: 535-560, 1990

Laurent M., Lepoivre M., and Tenu J.P. (1996) Kinetic modeling of the nitric oxide gradient generate in vitro by adherent cells expressing inducible nitric oxide synthase. Biochem J. 314: 109-113.

Palmer R.M.J., Ferrige A.G., Moncada S. (1987) Nitric oxide release accounts for the biological activity of endothelium-derived relaxing factor. Nature 327: 524-526.

Palmer R.M., Ashton D.S., Moncada S. (1988) Vascular endothelial cell synthesize nitric oxide from L-arginine. Nature 333: 664-666.

Rona G., Chapel C.I., Balazs T., Gaudry R. (1959) An infarct-like myocardial lesion and other toxic manifestations produced by isoproterenol on the rat. Arch Pathol 67:443-455.

Sander M, Chavoshan B. \& Victor R.G. (1999) A large blood pressure raising effect of nitric oxide synthase inhibition in humans, Hipertensión 33: 937-942.

Shultz PJ, Taych MA, Marletta MA, Raij L. (1991) Synthesis and action of nitric oxide in rats glomerular mesangial cells. Am J PHysiol 261: F600-F606.

Stanton H.C., Brenner G., Mayfield E.D. (1969) Studies on isoproterenol-induced cardiomegaly I rats. Am Heart J 77:72-80.

Suárez J., Torres C., Sánchez L., del Valle L. \& Pastelin G. (1999) Flow stimulates nitric oxide release in guinea pig heart. Role of stretch-activated ion channels. Biochem Biophys Res Comm, 261: 6-9.

Ueba Y., Ito Y., Mori K., Tomomatsu T. (1973) Studies of catecholamine metabolism in myocardial infarction. Singapore Medical J. 14: 351-352. 
Wallace A.C. and Klein R.F. (1969) Role of catecholamines in acute myocardial infarction. The Am J of Med Scs 258:139-143.

Wink D.A. \& Mitchell, J.B. (1998) Chemical biology of nitric oxide :Insights into regulatory, cytotoxic and citoprotective mechanisms of nitric oxide. Free Rad. Biol. Med 25:434456. 


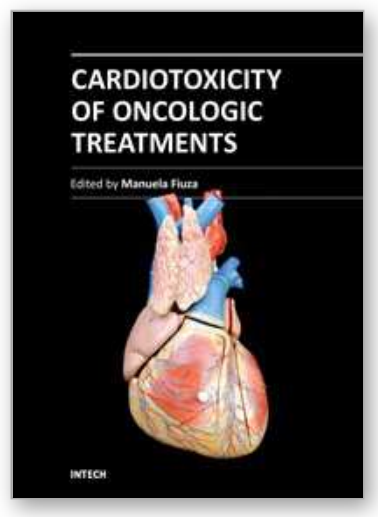

\section{Cardiotoxicity of Oncologic Treatments}

Edited by Prof. Manuela Fiuza

ISBN 978-953-51-0273-1

Hard cover, 194 pages

Publisher InTech

Published online 28, March, 2012

Published in print edition March, 2012

The possibility of getting a cardiovascular disease or cancer increases with advancing age. At the same time, relevant improvements in cancer therapy have resulted in the improvement of quality of life and the increasement of the survival rate of such patients. As a result we have larger number of patients that experience the cardiac side effects of chemotherapy. The extent of cardiotoxicity is variable, depending on the type of drug used, combination with other drugs, prior mediastinal radiotherapy and the presence of cardiovascular risk factors or history of heart disease. Early detection of the patients proneness for developing cardiotoxicity is the key issue to decrease morbidity and mortality. It also facilitates more tailored therapeutic interventions. Therefore, the collaboration and interaction of cardiology and oncology may contribute to reducing the cardiovascular adverse effects and improving the results in the treatment of patients with cancer.

\section{How to reference}

In order to correctly reference this scholarly work, feel free to copy and paste the following:

Victoria Chagoya de Sánchez, Lucía Yañez-Maldonado, Susana Vidrio-Gómez, Lidia Martínez, Jorge Suárez, Alberto Aranda-Fraustro, Juan Carlos Torres and Gabriela Velasco-Loyden (2012). Role of Nitric Oxide in Isoproterenol-Induced Myocardial Infarction, Cardiotoxicity of Oncologic Treatments, Prof. Manuela Fiuza (Ed.), ISBN: 978-953-51-0273-1, InTech, Available from: http://www.intechopen.com/books/cardiotoxicity-ofoncologic-treatments/role-of-nitric-oxide-in-isoproternol-induced-myocardial-infarction

\section{INTECH}

open science | open minds

\section{InTech Europe}

University Campus STeP Ri

Slavka Krautzeka 83/A

51000 Rijeka, Croatia

Phone: +385 (51) 770447

Fax: +385 (51) 686166

www.intechopen.com

\section{InTech China}

Unit 405, Office Block, Hotel Equatorial Shanghai

No.65, Yan An Road (West), Shanghai, 200040, China

中国上海市延安西路65号上海国际贵都大饭店办公楼 405 单元

Phone: +86-21-62489820

Fax: $+86-21-62489821$ 
(C) 2012 The Author(s). Licensee IntechOpen. This is an open access article distributed under the terms of the Creative Commons Attribution 3.0 License, which permits unrestricted use, distribution, and reproduction in any medium, provided the original work is properly cited. 